\title{
Review of numerical methods for simulation of mechanical heart valves and the potential for blood clotting
}

\begin{abstract}
Even though the mechanical heart valve (MHV) has been used routinely in clinical practice for over 60 years, the occurrence of serious complications such as blood clotting remains to be elucidated. This paper reviews the progress that has been made over the years in terms of numerical simulation method and the contribution of abnormal flow toward blood clotting from MHVs in the aortic position. It is believed that this review would likely be of interest to some readers in various disciplines, such as engineers, scientists, mathematicians and surgeons, to understand the phenomenon of blood clotting in MHVs through computational fluid dynamics.
\end{abstract}

Keyword: Computational fluid dynamics; Mechanical heart valve; Blood clot; Cartesian mesh 